\title{
Baseline Urinary Angiotensinogen Excretion Predicts Deterioration of the Kidney Function in Patients with Chronic Kidney Disease
}

\author{
Sayaka Ishigaki ${ }^{1}$, Naro Ohashi ${ }^{2}$, Taro Aoki ${ }^{2}$, Takashi Matsuyama ${ }^{2}$, Shinsuke Isobe ${ }^{2}$, \\ Taichi Sato $^{2}$, Tomoyuki Fujikura ${ }^{2}$, Akihiko Kato ${ }^{1}$ and Hideo Yasuda ${ }^{2}$
}

\begin{abstract}
:
Objective The intrarenal renin-angiotensin system (RAS) is activated in patients with chronic kidney disease (CKD), and urinary angiotensinogen (AGT) levels, a surrogate marker of the intrarenal RAS activation, are associated with blood pressure (BP) and urinary albumin excretion. In addition, it has been shown that changes in urinary AGT levels correlate with annual changes in the estimated glomerular filtration rate (eGFR) in patients with type 2 diabetes and that elevated levels of urinary AGT in type 2 diabetic patients with albuminuria are a high-risk factor for worsening renal and cardiovascular complications. However, whether or not baseline urinary AGT levels predict deterioration of the kidney function in all patients with CKD is unclear.

Methods We recruited 62 patients with CKD whose eGFR was $>15 \mathrm{~mL} / \mathrm{min} / 1.73 \mathrm{~m}^{2}$. We performed 24hour ambulatory BP monitoring at 30-min intervals and daily urinary collection to examine the urinary AGT levels and albumin excretion and measured the levels of plasma angiotensin II (Ang II), a surrogate marker of circulating RAS. In addition, annual changes in the eGFR were followed up for $3.4 \pm 1.5$ years.

Results Annual changes in the eGFR were significantly and negatively associated with urinary AGT levels $(\mathrm{r}=-0.31, \mathrm{p}=0.015)$ as well as the age, systolic BP, and urinary albumin levels. In contrast, annual changes in the eGFR were not correlated with plasma Ang II levels. Furthermore, when dividing patients into quartiles according to urinary AGT levels, patients with the highest urinary AGT levels showed a progressive decline in the eGFR.

Conclusion These results suggest that elevated baseline urinary AGT levels can predict renal dysfunction in patients with CKD.
\end{abstract}

Key words: chronic kidney disease, intrarenal renin-angiotensin system, renal prognosis, urinary angiotensinogen

(Intern Med 60: 2201-2206, 2021)

(DOI: 10.2169/internalmedicine.6599-20)

\section{Introduction}

The circulating renin-angiotensin system (RAS) plays a critical role in the regulation of arterial pressure and sodium homeostasis (1). A tissue-specific RAS, independent of the circulating RAS, has been characterized in several organs. Researchers have reported that the intrarenal RAS is acti- vated in some animal models and patients with chronic kidney disease (CKD) or hypertension and that activation of the intrarenal RAS is intimately involved in the pathophysiology of renal damage (2-6).

Angiotensinogen (AGT) is the only known substrate for renin, the rate-limiting enzyme in the RAS. AGT levels influence RAS activation since they are close to the Michaelis-Menten constant for renin $(7,8)$, and urinary AGT

${ }^{1}$ Blood Purification Unit, Hamamatsu University School of Medicine, Japan and ${ }^{2}$ Internal Medicine 1, Hamamatsu University School of Medicine, Japan

Received: October 27, 2020; Accepted: January 7, 2021; Advance Publication by J-STAGE: February 22, 2021

Correspondence to Dr. Naro Ohashi, ohashi-n@hama-med.ac.jp 


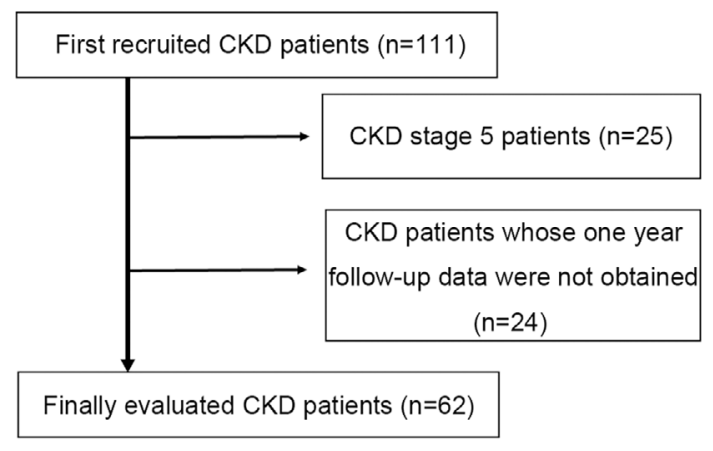

Figure 1. Recruitment and enrollment of this study. We consecutively recruited 111 patients with chronic kidney disease (CKD) 20-80 years old who were admitted to our hospital and whose urinary angiotensinogen (AGT) excretion had been measured between January 2012 and December 2016. We excluded 25 patients with CKD whose estimated glomerular filtration rate (eGFR) was $<15 \mathrm{~mL} / \mathrm{min} / 1.73 \mathrm{~m}^{2}$ (CKD stage 5). In addition, we excluded 24 patients with CKD whose one-year follow-up data were not available. Finally, we evaluated 62 patients with CKD in this study.

is reported to be a useful biomarker that reflects the intrarenal RAS activity and CKD severity (2, 5, 6, 9-13).

Recently, Lee et al. recruited 91 patients with type 2 diabetes who were followed up for 52 months and found that changes in urinary AGT correlated with a decline in the kidney function (14). In addition, Sawaguchi et al. reported that elevated levels of urinary AGT in type 2 diabetic patients with albuminuria were a risk factor for worsening renal and cardiovascular complications (15). However, whether or not the baseline urinary AGT levels predict deterioration of the kidney function in all CKD patients, irrespective of the cause of $\mathrm{CKD}$, is unclear.

Therefore, in the present study, we examined the relationships between baseline urinary AGT levels and annual changes in the estimated glomerular filtration rate (eGFR) in quartiles according to baseline urinary AGT levels.

\section{Materials and Methods}

\section{Patients}

This study was approved by the ethics committee of Hamamatsu University School of Medicine and adhered to the principles of the Declaration of Helsinki. We consecutively recruited 111 patients with CKD 20-80 years old who were admitted to our hospital for close investigation by a renal biopsy and whose baseline urinary AGT excretion had been measured to evaluate the RAS function in the kidney between January 2012 and December 2016. We excluded 25 patients with CKD whose eGFR was $<15 \mathrm{~mL} / \mathrm{min} / 1.73 \mathrm{~m}^{2}$ (CKD stage 5) because those patients were introduced to dialysis or underwent a kidney transplant within less than 1 year and annual follow-up was not expected. In addition, we excluded 24 patients with CKD whose 1-year follow-up data were not obtained for reasons such as switching hospitals. We ultimately evaluated 62 patients with CKD in this study (Fig. 1). The patients were followed up annually in our outpatient department until December 2018. Written informed consent was obtained from all patients.

\section{Study protocols}

On admission, ambulatory blood pressure monitoring (ABPM) using an automatic device (TM-2431; A and D, Tokyo, Japan) was carried out for 24 hours with 30-min intervals, and blood samples were collected at 6:00 AM at the end of the ABPM, after the patients with CKD had rested in the supine position for at least 15 minutes. Urine samples were also obtained all day long on the day the ABPM was performed. The blood samples were centrifuged at 3,000 $\mathrm{rpm}$ at $4^{\circ} \mathrm{C}$ for 10 minutes, while the urine samples were centrifuged at $1,500 \mathrm{rpm}$ at $4^{\circ} \mathrm{C}$ for 5 minutes. Both samples were stored at $-80^{\circ} \mathrm{C}$ until assays were performed. These experiments were performed as described previously $(2,16-18)$. Thereafter, the patients were followed up annually at our outpatient department.

\section{Clinical data}

The patients' clinical data, including their age, sex, and body mass index (BMI), were recorded at the time of admission. During 24-hour ABPM, the BP was measured noninvasively every 30 minutes, as described above. Serum and urinary creatinine concentrations were measured in the clinical laboratory of the Hamamatsu University School of Medicine, University Hospital. The levels of urinary AGT, which is known to be a surrogate marker of the intrarenal RAS activity $(2,5,6,9-13)$, were measured using an enzyme-linked immunosorbent assay as described previously (19). Urinary albumin concentrations and plasma angiotensin II (Ang II) levels were determined using a radioimmunoassay (SRL, Tokyo, Japan). Serum creatinine concentrations were measured in blood drawn at 6:00 AM, and the eGFR was calculated using the serum creatinine concentrations in the Japanese eGFR equation (20). The excretion ratios of urinary AGT/ creatinine (AGT/Cr) were calculated. The annual rate of change in the eGFR $\left(\mathrm{mL} / \mathrm{min} / 1.73 \mathrm{~m}^{2} /\right.$ year) was determined from the slope calculated by a linear regression analysis of the eGFR measured for each individual annually during follow-up, as described previously $(14,15)$.

\section{Statistical analyses}

The results are expressed as mean \pm standard deviation. The Shapiro-Wilk test was performed to examine whether or not the variables were normally distributed. Because the levels of daily urinary albumin excretion and urinary AGT/Cr were not normally distributed, logarithmic transformation was applied. The correlations between the annual change in the eGFR and the age, sex, BMI, systolic and diastolic BPs, heart rate, and baseline levels of the eGFR, daily urinary albumin excretion, plasma Ang II, and urinary AGT/Cr on admission were evaluated using Pearson's product-moment 
Table 1. Patient Characteristics.

\begin{tabular}{|c|c|}
\hline Age (year) & $48.5 \pm 17.7$ \\
\hline Sex & Male 25 / Female 37 \\
\hline Causes of CKD & DKD: 2 / CGN: 47 / NS: 3 / Other 10 \\
\hline RAS blocker usage & $\begin{array}{l}\text { at the start of the study: } 17 \text { (ARB: } 16, \text { ACE-I: } 1) \\
\text { during the study: } 35 \text { (ARB: } 33 \text {, ACE-I: } 2 \text { ) }\end{array}$ \\
\hline Height $(\mathrm{cm})$ & $161.7 \pm 9.4$ \\
\hline Body weight $(\mathrm{kg})$ & $55.7 \pm 10.6$ \\
\hline Body mass index $\left(\mathrm{kg} / \mathrm{m}^{2}\right)$ & $21.2 \pm 3.0$ \\
\hline Systolic BP (mmHg) & $118.5 \pm 14.5$ \\
\hline Diastolic BP (mmHg) & $72.3 \pm 9.2$ \\
\hline Heart rate $(/ \mathrm{min})$ & $66.1 \pm 7.5$ \\
\hline $\mathrm{sCr}(\mathrm{mg} / \mathrm{dL})$ & $1.05 \pm 0.45$ \\
\hline $\mathrm{eGFR}\left(\mathrm{mL} / \mathrm{min} / 1.73 \mathrm{~m}^{2}\right)$ & $59.8 \pm 22.6$ \\
\hline CKD stage & $\begin{array}{c}\text { Stage 1: } 8 \text { / Stage 2: } 22 \text { / Stage 3a: 17/ Stage 3b: } 11 \text { / } \\
\text { Stage 4: } 4 \text { / Stage 5: } 0\end{array}$ \\
\hline Plasma angiotensin II (pg/mL) & $13.1 \pm 10.0$ \\
\hline Log urinary albumin (mg/day) & $2.42 \pm 0.60$ \\
\hline Log urinary AGT/Cr $(\mu \mathrm{g} / \mathrm{gCr})$ & $1.87 \pm 0.60$ \\
\hline
\end{tabular}

correlation test. Multiple linear regression analyses were conducted to evaluate the relationships between the annual change in the eGFR and the baseline urinary AGT/Cr levels. The age, sex, BMI, and baseline eGFR were selected as independent variables, as these parameters were commonly included in multiple linear regression analyses.

We then divided all patients into quartiles according to the baseline urinary AGT/Cr excretion. Thereafter, a comparison among these four groups was performed by an analysis of variance (ANOVA) with the Tukey-Kramer HSD test or Games Howell test. Covariance analyses were performed to examine the association between the quartiles of the baseline urinary AGT/Cr excretion and annual change in the eGFR adjusted for the age, sex, BMI, and baseline eGFR. We considered values of $p<0.05$ to indicate statistical significance. Statistical analyses were performed using the IBM $^{\circledR}$ SPSS $^{\circledR}$ software program, version 25 (IBM, Armonk, USA).

\section{Results}

\section{Patient characteristics}

Sixty-two patients with CKD who were admitted to our hospital during the study duration were included in this study. Their baseline characteristics are presented in Table 1. Due to most patients having been admitted to undergo a renal biopsy for chronic glomerulonephritis, most patients were middle-aged $(48.5 \pm 17.7$ years old), and their renal function was preserved (serum creatinine: $1.05 \pm 0.45 \mathrm{mg} / \mathrm{dL}$; eGFR: $59.8 \pm 22.6 \mathrm{~mL} / \mathrm{min} / 1.73 \mathrm{~m}^{2}$ ), with logarithmic urinary albumin excretion of $2.42 \pm 0.60 \mathrm{mg} /$ day. The number of pa- tients administered RAS blockers was 17 [Ang II receptor blockers (ARBs), n=16; angiotensin-converting enzyme inhibitors (ACE-Is), $n=1]$ at the start of this study and 35 (ARBs, n=33; ACE-Is, n=2) over the course of this study.

\section{Annual change in the eGFR in all patients}

The average follow-up period was $3.4 \pm 1.5$ years, and the average annual change in the eGFR was $-0.93 \pm 6.16 \mathrm{~mL} /$ $\mathrm{min} / 1.73 \mathrm{~m}^{2}$ during this period.

\section{Relationship between the annual change in the eGFR and several clinical parameters, including the baseline urinary AGT excretion}

We first evaluated the relationship between the annual change in the eGFR and several clinical parameters, including baseline urinary AGT excretion. Significant negative relationships were found between the annual change in the eGFR and the age $(\mathrm{r}=-0.35, \mathrm{p}<0.01)$, systolic BP $(\mathrm{r}=-0.36, \mathrm{p}$ $<0.01)$ and daily urinary albumin excretion ( $\mathrm{r}=-0.32, \mathrm{p}=$ 0.011) (Table 2). In addition, the annual change in the eGFR was significantly and negatively correlated with the baseline urinary AGT excretion ( $\mathrm{r}=-0.31, \mathrm{p}=0.015$ ) (Fig. 2). However, no significant relationships were found between the annual change in the eGFR and plasma Ang II $(r=0.22, \mathrm{p}=0.10)$ (Table 2). We also performed multiple linear regression analyses between the annual change in the eGFR and baseline urinary AGT excretion after adjusting for the age, sex, BMI, and baseline eGFR. A significant negative relationship was found between them after adjusting in this manner $(\beta=-$ 0.27, $\mathrm{p}=0.032$ ) (Table 3). 
Table 2. Relationship between Annual Change in Estimated Glomerular Filtration Rate (eGFR) and Some Clinical Parameters.

\begin{tabular}{lcc}
\hline & $\mathrm{r}$ & $\mathrm{p}$ \\
\hline Age (year) & -0.35 & $<0.01$ \\
Body mass index $\left(\mathrm{kg} / \mathrm{m}^{2}\right)$ & -0.086 & 0.51 \\
Systolic BP $(\mathrm{mmHg})$ & -0.36 & $<0.01$ \\
Diastolic BP $(\mathrm{mmHg})$ & -0.19 & 0.14 \\
Heart rate $(/ \mathrm{min})$ & 0.19 & 0.13 \\
Baseline eGFR $\left(\mathrm{mL} / \mathrm{min} / 1.73 \mathrm{~m}^{2}\right)$ & -0.032 & 0.80 \\
Log urinary albumin/day $(\mathrm{mg} /$ day $)$ & -0.32 & 0.011 \\
Plasma Ang II $(\mathrm{pg} / \mathrm{mL})$ & 0.22 & 0.10 \\
\hline BP: blood pressure, Log: logarithmic, Ang II: angiotensin II
\end{tabular}

Table 3. Multiple Linear Regression Analyses between Annual Change in Estimated Glomerular Filtration Rate (eGFR) and Baseline Urinary Angiotensinogen (AGT) Excretion Levels after Adjustment for Age, Sex, Body Mass Index (BMI) and Baseline eGFR.

\begin{tabular}{lcc}
\hline & $\mathrm{r}=0.52$ & $\mathrm{p}<0.01$ \\
\hline Age (year) & $\beta$ & $\mathrm{p}$ \\
Sex & -0.45 & $<0.01$ \\
BMI & -0.10 & 0.40 \\
Baseline eGFR $\left(\mathrm{mL} / \mathrm{min} / 1.73 \mathrm{~m}^{2}\right)$ & -0.04 & 0.75 \\
Log urinary AGT/Cr $(\mu \mathrm{g} / \mathrm{g})$ & -0.37 & 0.011 \\
\hline
\end{tabular}

Log: logarithmic, Cr: creatinine

The comparison of the annual change in the eGFR among quartiles according to the baseline urinary AGT excretion

We then divided the patients into quartiles according to the baseline urinary AGT excretion and compared the levels of clinical parameters among the quartiles. The systolic and diastolic BPs in the highest quartile of baseline urinary AGT excretion (Group 4) (systolic BP: $124.9 \pm 12.7 \mathrm{mmHg}$ and diastolic BP: $77.5 \pm 10.4 \mathrm{mmHg}$ ) were significantly higher than those in Group 1 (systolic BP: $109.2 \pm 9.9 \mathrm{mmHg}$; $\mathrm{p}<$ 0.05 and diastolic BP: $66.3 \pm 4.6 \mathrm{mmHg} ; \mathrm{p}<0.01)$. In addition, the logarithmic daily urinary albumin excretion $(2.99 \pm$ $0.31 \mathrm{mg} /$ day) in the highest quartile (Group 4) was higher than that in the other groups (Group 1: $1.98 \pm 0.43 \mathrm{mg} / \mathrm{day}$; $\mathrm{p}<0.05$, Group 2: $2.43 \pm 0.55 \mathrm{mg} / \mathrm{day} ; \mathrm{p}<0.05$, and Group 3: $2.34 \pm 0.63 \mathrm{mg} /$ day; $\mathrm{p}<0.05$ ) (Supplementary material 1 ). The annual change in the eGFR in the highest quartile of baseline urinary AGT excretion (Group 4; $-5.48 \pm 7.14 \mathrm{~mL} / \mathrm{min} /$ $1.73 \mathrm{~m}^{2} /$ year) was significantly lower than that in Group 2 $\left(1.41 \pm 3.39 \mathrm{~mL} / \mathrm{min} / 1.73 \mathrm{~m}^{2} / \mathrm{year} ; \mathrm{p}<0.01\right)$ and Group 3 $\left(0.46 \pm 5.50 \mathrm{~mL} / \mathrm{min} / 1.73 \mathrm{~m}^{2} /\right.$ year; $\left.\mathrm{p}=0.023\right)$. In addition, a similar tendency was found between the lowest quartile of baseline urinary AGT excretion (Group 1: $-0.31 \pm 6.11 \mathrm{~mL} /$ $\min / 1.73 \mathrm{~m}^{2} /$ year) and Group 4 ( $\mathrm{p}=0.073$ ) (Fig. 2).

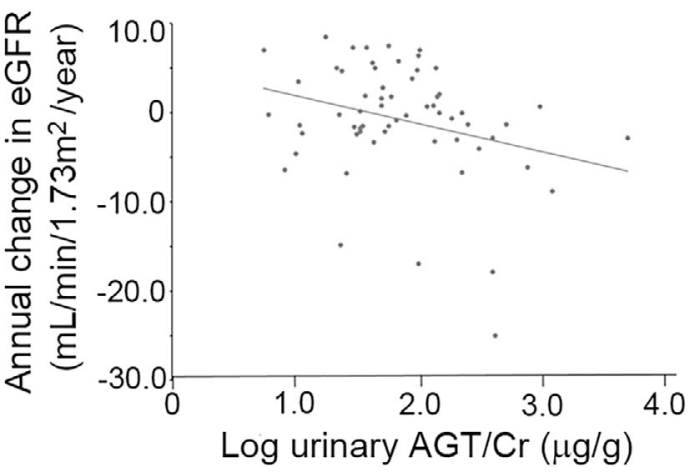

Figure 2. The relationship between the annual change in the estimated glomerular filtration rate (eGFR) and baseline urinary angiotensinogen (AGT) excretion. The annual change in the eGFR was significantly and negatively correlated with the urinary AGT excretion $(r=-0.31, p=0.015)$.

Covariance analyses between the quartiles of baseline urinary AGT excretion and annual change in the eGFR after adjustment

Covariance analyses were also performed to examine the association between the quartiles of the baseline urinary AGT excretion and the annual change in the eGFR adjusted for the age, sex, BMI and baseline eGFR. Covariance analyses showed that the quartiles of baseline urinary AGT excretion differed significantly with regard to the annual change in the eGFR after adjustment (Model 1: Group 1 vs. Group 4, $\mathrm{p}=0.11$; Group 2 vs. Group 4, $\mathrm{p}<0.01$; and Group 3 vs. Group 4, $\mathrm{p}=0.011$; and Model 2: Group 1 vs. Group 4, $\mathrm{p}=$ 0.09 ; Group 2 vs. Group 4, $\mathrm{p}<0.01$; and Group 3 vs. Group 4, p=0.031) (Fig. 3 and Table 4).

\section{Discussion}

This study showed that the annual change in the eGFR was significantly and negatively associated with the baseline urinary AGT levels, even after adjusting for several factors. In addition, when the baseline urinary AGT levels were divided into quartiles, the patients with $\mathrm{CKD}$ with the highest baseline urinary AGT levels showed a progressive decline in the eGFR compared with the patients with lower baseline urinary AGT levels, and covariance analyses showed that the quartiles of the baseline urinary AGT excretion differed significantly with respect to the annual change in the eGFR after adjustment. These results suggest that elevated urinary AGT levels predict renal dysfunction in patients with CKD.

Hypertension is associated with an increased risk of development of CKD. Kanno et al. examined 2,150 individuals without preexisting CKD from the general population during a mean follow-up of 6.5 years, and 461 incidences of CKD were recorded. They indicated that the adjusted hazard ratios of $\mathrm{CKD}$ were significantly higher for pre-hypertension $(1.49, \mathrm{p}<0.003)$, Stage $1(1.83, \mathrm{p}<0.001)$ and Stage 2 (2.55, $\mathrm{p}<0.001)$ hypertension in the study than normotension (21). 


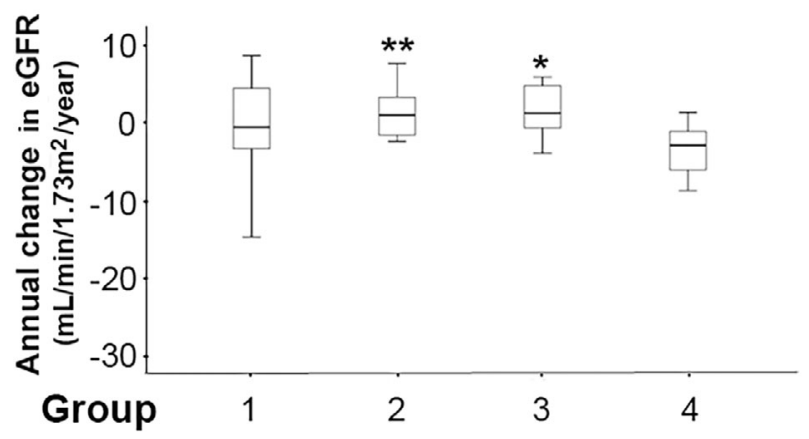

Figure 3. The comparison of the annual change in the estimated glomerular filtration rate (eGFR) among quartiles according to the baseline urinary angiotensinogen (AGT) excretion. The patients were divided into the quartiles according to the baseline urinary AGT excretion, and the levels of annual change in the eGFR were compared among the quartiles. Box plots represent the 25th percentile, median, and 75th percentile of each group. Error bars denote the 10th and 90th percentiles. Groups were numbered from the lowest quartile of baseline urinary AGT excretion. Data are means \pm standard deviation. **p $<0.01$ Group 2 vs. Group 4. *p<0.05 Group 3 vs. Group 4.

In contrast, Kiriyama et al. examined 2,739 individuals who underwent repeated health checkups, and they found that an eGFR decline was more commonly observed in individuals with proteinuria at baseline than in those without proteinuria at baseline (individuals with proteinuria: $3.3 \%$ vs. individuals without proteinuria: $0.8 \%, \mathrm{p}<0.001)(22)$. These previous reports coincide with our data indicating that systolic BP and urinary albumin excretion were predictors of renal dysfunction in the present study. Furthermore, it has also been demonstrated that urinary AGT is a surrogate marker of intrarenal RAS activity $(2,5,6,9-13)$ and that urinary AGT is associated with the levels of renal damage and BPs (2-6). Therefore, we suspect that the baseline urinary AGT levels predicted renal dysfunction in the present study.

It may not be meaningful to measure urinary AGT levels, as urinary AGT levels may serve as a replacement for renal damage or hypertension. However, we reported that systolic BP progressively increased in double transgenic mice expressing human renin systemically in addition to human AGT in the kidney (23). Saito et al. indicated that an increase in urinary AGT levels preceded an increase in urinary albumin levels in patients with type 1 diabetes (11). We previously indicated that the intrarenal RAS is activated in kidney transplant donors immediately after kidney donation, prior to an increase in urinary albumin levels (24). These findings indicate that intrarenal RAS activation induces renal damage, such as microalbuminuria and hypertension. Therefore, urinary AGT levels are not merely reflective of renal damage and hypertension; it is meaningful to measure urinary AGT levels.

Recently, Lee et al. reported that changes in urinary AGT correlated with a decline in the kidney function in patients with type 2 diabetes (14), and Sawaguchi et al. reported that
Table 4. Covariance Analyses to Determine the Association between the Quartiles of Baseline Urinary Angiotensinogen (AGT) Excretion Levels and Annual Change in Estimated Glomerular Filtration Rate (eGFR) after Adjustment for Age, Sex, Body Mass Index (BMI) and Baseline eGFR.

\begin{tabular}{lcccccc}
\hline & \multicolumn{2}{c}{ Model 1 } & & \multicolumn{2}{c}{ Model 2 } \\
\cline { 2 - 3 } \cline { 5 - 6 } & $\mathrm{F}$ & $\mathrm{p}$ & & $\mathrm{F}$ & $\mathrm{p}$ \\
\hline Age (year) & 6.51 & 0.014 & & 11.05 & $<0.01$ \\
Sex & 0.65 & 0.42 & & 1.21 & 0.28 \\
BMI $\left(\mathrm{kg} / \mathrm{m}^{2}\right)$ & 0.16 & 0.69 & & 0.22 & 0.64 \\
Baseline eGFR $\left(\mathrm{mL} / \mathrm{min} / 1.73 \mathrm{~m}^{2}\right)$ & & & & 4.33 & 0.042 \\
Log urinary AGT/Cr $(\mu \mathrm{g} / \mathrm{gCr})$ & 4.32 & $<0.01$ & & 3.98 & 0.013 \\
\hline
\end{tabular}

BMI: body mass index, Log: logarithmic, Cr: creatinine

elevated levels of urinary AGT in type 2 diabetic patients with albuminuria were a risk factor for worsening renal and cardiovascular complications (15). In addition, we previously indicated that intrarenal RAS activation was significantly and positively correlated with renal damage and hypertension in patients with CKD, including diabetic nephropathy patients (2). This suggests that baseline urinary AGT levels predicted deterioration of the kidney function in all patients with CKD in the present study. However, the AGT expression in glomerular mesangial cells is reportedly increased by high glucose levels $(25,26)$. Furthermore, the AGT expression in the proximal tubular cells is stimulated by high glucose levels. Immediately after a sodium-glucose co-transporter 2 (SGLT2) inhibitor is administered, urinary AGT levels are increased by increases in the glucose levels in the proximal tubular lumen. However, when glucose levels are decreased by an SGLT2 inhibitor, the glucose levels in the proximal tubular lumen decrease, as does the AGT expression in the proximal tubular cells (27). As mentioned previously, the degree of intrarenal RAS activation differs among certain conditions, such as based on the glucose levels and prescription drug use. Therefore, it was possible that the results of all patients with CKD in the present study are different from those of only patients with diabetes in the previous studies. However, we obtained results that were similar to those in the previous studies, suggesting that urinary AGT levels predict renal dysfunction in the present study.

The patients with RAS blockers showed elevated values of systolic BP (with RAS blockers: 124.7 $\pm 15.0 \mathrm{mmHg}$ vs. without RAS blockers: $110.4 \pm 9.0 \mathrm{mmHg}$; $\mathrm{p}<0.01$ ), urinary Alb excretion (with RAS blockers: $2.63 \pm 0.55 \mathrm{mg} /$ day vs. without RAS blockers: 2.22 \pm 0.58 ; $\mathrm{p}=0.014$ ), and baseline urinary AGT excretion (with RAS blockers: 2.02 $\pm 0.57 \mu \mathrm{g} /$ $\mathrm{gCr}$ vs. without RAS blockers: $1.67 \pm 0.60 \mu \mathrm{g} / \mathrm{gCr}$; $\mathrm{p}=0.024)$. Furthermore, the annual decline in the eGFR with RAS blockers was greater than that without RAS blockers (with RAS blockers: $-3.08 \pm 6.85 \mathrm{~mL} / \mathrm{min} / 1.73 \mathrm{~m}^{2}$ vs. without RAS blockers: $1.68 \pm 3.94 \mathrm{~mL} / \mathrm{min} / 1.73 \mathrm{~m}^{2} ; \mathrm{p}<0.01$ ) (data not shown). These results suggest that RAS blockers were administered to patients with relatively severe CKD in our 
study.

Several limitations associated with the present study warrant mention. First, its sample size was small, and patients were recruited from a single center. Second, the follow-up period was $3.4 \pm 1.5$ years, and the duration was relatively short. Finally, although some interventions with diets, such as a low-sodium diet, were made during the follow-up period in our outpatient department, the interventions were not equal for all patients with CKD. In addition, the salt intake was not evaluated by the collection of daily urine for all patients. Therefore, it was difficult for us to evaluate the influence of food intake on the findings. Nevertheless, we were able to demonstrate that patients with CKD with elevated baseline urinary AGT levels, similar to those with elevated urinary albumin levels and BP values, showed rapid renal dysfunction compared with other patients.

In conclusion, the annual change in the eGFR was significantly and negatively associated with the baseline urinary AGT levels. Furthermore, patients in the highest quartile of baseline urinary AGT levels revealed a progressive decline in the eGFR. These results suggest that elevated baseline urinary AGT levels predict rapid renal dysfunction in patients with CKD. In the future, larger and longer-term studies will be required to further our findings.

The authors state that they have no Conflict of Interest (COI).

\section{References}

1. Kobori H, Nangaku M, Navar LG, Nishiyama A. The intrarenal renin-angiotensin system: from physiology to the pathobiology of hypertension and kidney disease. Pharmacol Rev 59: 251-287, 2007.

2. Isobe S, Ohashi N, Fujikura T, et al. Disturbed circadian rhythm of the intrarenal renin-angiotensin system: relevant to nocturnal hypertension and renal damage. Clin Exp Nephrol 19: 231-239, 2015.

3. Ohashi N, Katsurada A, Miyata K, et al. Activation of reactive oxygen species and the renin-angiotensin system in IgA nephropathy model mice. Clin Exp Pharmacol Physiol 36: 509-515, 2009.

4. Isobe $\mathrm{S}$, Ohashi N, Ishigaki S, et al. Augmented circadian rhythm of the intrarenal renin-angiotensin systems in anti-thymocyte serum nephritis rats. Hypertens Res 39: 312-320, 2016.

5. Kobori H, Alper AB Jr, Shenava R, et al. Urinary angiotensinogen as a novel biomarker of the intrarenal renin-angiotensin system status in hypertensive patients. Hypertension 53: 344-350, 2009.

6. Kobori H, Ohashi N, Katsurada A, et al. Urinary angiotensinogen as a potential biomarker of severity of chronic kidney diseases. J Am Soc Hypertens 2: 349-354, 2008.

7. Gould AB, Green D. Kinetics of the human renin and human substrate reaction. Cardiovasc Res 5: 86-89, 1971.

8. Brasier AR, Li J. Mechanisms for inducible control of angiotensinogen gene transcription. Hypertension 27: 465-475, 1996.

9. Yamamoto T, Nakagawa T, Suzuki H, et al. Urinary angiotensinogen as a marker of intrarenal angiotensin II activity associated with deterioration of renal function in patients with chronic kidney disease. J Am Soc Nephrol 18: 1558-1565, 2007.

10. Nishiyama A, Konishi Y, Ohashi N, et al. Urinary angiotensinogen reflects the activity of intrarenal renin-angiotensin system in pa- tients with IgA nephropathy. Nephrol Dial Transplant 26: 170-177, 2011.

11. Saito T, Urushihara M, Kotani Y, Kagami S, Kobori H. Increased urinary angiotensinogen is precedent to increased urinary albumin in patients with type 1 diabetes. Am J Med Sci 338: 478-480, 2009.

12. Kobori H, Harrison-Bernard LM, Navar LG. Urinary excretion of angiotensinogen reflects intrarenal angiotensinogen production. Kidney Int 61: 579-585, 2002.

13. Kobori H, Navar LG. Urinary angiotensinogen as a novel biomarker of intrarenal renin-angiotensin system in chronic kidney disease. Int Rev Thromb 6: 108-116, 2011.

14. Lee MJ, Kim SS, Kim IJ, et al. Changes in urinary angiotensinogen associated with deterioration of kidney function in patients with type 2 diabetes mellitus. J Korean Med Sci 32: 782-788, 2017.

15. Sawaguchi M, Araki SI, Kobori H, et al. Association between urinary angiotensinogen levels and renal and cardiovascular prognoses in patients with type 2 diabetes mellitus. J Diabetes Investig $\mathbf{3}$ : 318-324, 2012.

16. Ishigaki $\mathrm{S}$, Ohashi $\mathrm{N}$, Isobe $\mathrm{S}$, et al. Impaired endogenous nighttime melatonin secretion relates to intrarenal renin-angiotensin system activation and renal damage in patients with chronic kidney disease. Clin Exp Nephrol 20: 878-884, 2016.

17. Ohashi $\mathrm{N}$, Isobe $\mathrm{S}$, Ishigaki $\mathrm{S}$, et al. The effects of unilateral nephrectomy on blood pressure and its circadian rhythm. Intern Med 55: 3427-3433, 2016.

18. Fukuda M, Mizuno M, Yamanaka T, et al. Patients with renal dysfunction require a longer duration until blood pressure dips during the night. Hypertension 52: 1155-1160, 2008.

19. Katsurada A, Hagiwara $Y$, Miyashita $K$, et al. Novel sandwich ELISA for human angiotensinogen. Am J Physiol Renal Physiol 293: F956-F960, 2007.

20. Matsuo S, Imai E, Horio M, et al. Collaborators developing the Japanese equation for estimated GFR. Collaborators developing the Japanese equation for estimated GFR. Revised equations for estimated GFR from serum creatinine in Japan. Am J Kidney Dis 53: 982-992, 2009

21. Kanno A, Kikuya M, Ohkubo T, et al. Pre-hypertension as a significant predictor of chronic kidney disease in a general population: the Ohasama Study. Nephrol Dial Transplant 27: 3218-3223, 2012.

22. Kiriyama H, Kaneko H, Itoh H, et al. Role of anemia and proteinuria in the development of subsequent renal function deterioration in a general population with preserved glomerular filtration rate: a community-based cohort study. J Nephrol 32: 775-781, 2019.

23. Kobori H, Ozawa Y, Satou R, et al. Kidney-specific enhancement of ANG II stimulates endogenous intrarenal angiotensinogen in gene-targeted mice. Am J Physiol Renal Physiol 293: F938-F945, 2007.

24. Ohashi $\mathrm{N}$, Isobe $\mathrm{S}$, Matsuyama $\mathrm{T}$, et al. The intrarenal reninangiotensin system is activated immediately after kidney donation in kidney transplant donors. Intern Med 58: 643-648, 2019.

25. Singh R, Singh AK, Alavi N, Leehey DJ. Mechanism of increased angiotensin II levels in glomerular mesangial cells cultured in high glucose. J Am Soc Nephrol 14: 873-880, 2003.

26. Vidotti DB, Casarini DE, Cristovam PC, Leite CA, Schor N, Boim MA. High glucose concentration stimulates intracellular renin activity and angiotensin II generation in rat mesangial cells. Am J Physiol Renal Physiol 286: F1039-F1045, 2004.

27. Shin SJ, Chung S, Kim SJ, et al. Effect of sodium-glucose cotransporter 2 inhibitor, dapagliflozin, on renal renin-angiotensin system in an animal model of type 2 diabetes. PLoS One 11: e0165703, 2016

The Internal Medicine is an Open Access journal distributed under the Creative Commons Attribution-NonCommercial-NoDerivatives 4.0 International License. To view the details of this license, please visit (https://creativecommons.org/licenses/ by-nc-nd/4.0/).

(C) 2021 The Japanese Society of Internal Medicine Intern Med 60: 2201-2206, 2021 